\title{
Design of Conformationally Distorted Donor-Acceptor Dyads Showing Efficient Thermally Activated Delayed Fluorescence
}

Gereon A. Sommer, ${ }^{\mathrm{a}}$ Larisa N. Mataranga-Popa, ${ }^{\mathrm{b}}$ Rafal Czerwieniec, ${ }^{\mathrm{b}}$ Thomas Hofbeck, ${ }^{\mathrm{b}}$

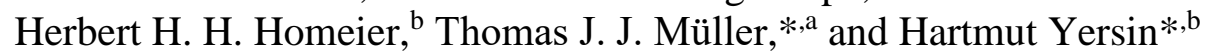

${ }^{a}$ Institut für Organische Chemie und Makromolekulare Chemie, Heinrich-Heine-Universität Düsseldorf, Universitätsstrasse 1, D-40225 Düsseldorf, Germany

${ }^{b}$ Institut für Physikalische Chemie, Universität Regensburg, Universitätsstr. 31, D-93053 Regensburg, Germany

J. Phys. Chem. Lett. 2018, 9, 3692-3697

DOI: $10.1021 /$ acs.jpclett.8b01511

This project has received funding from the European Union's Horizon 2020 research and innovation programme under the Marie Skłodowska-Curie grant agreement No. 645628

Project Number: 645628

Project Acronym: METCOPH

Project title: Metallocomplexes of macrocyclic compounds for photonic devices

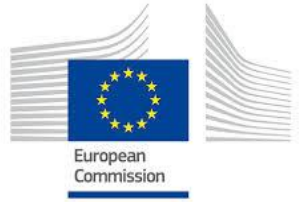




\section{Design of Conformationally Distorted Donor-Acceptor Dyads Showing Efficient Thermally Activated Delayed Fluorescence}

Gereon A. Sommer, ${ }^{a}$ Larisa N. Mataranga-Popa, ${ }^{\mathrm{b}}$ Rafal Czerwieniec, ${ }^{\mathrm{b}}$ Thomas Hofbeck, ${ }^{\mathrm{b}}$ Herbert H. H. Homeier, ${ }^{b}$ Thomas J. J. Müller, ${ }^{*, a}$ and Hartmut Yersin ${ }^{*, b}$

aInstitut für Organische Chemie und Makromolekulare Chemie, Heinrich-Heine-Universität Düsseldorf, Universitätsstrasse 1, D-40225 Düsseldorf, Germany

'Institut für Physikalische Chemie, Universität Regensburg, Universitätsstr. 31, D-93053 Regensburg, Germany

Email: ThomasJJ.Mueller@hhu.de; $\underline{\text { hartmut.yersin@ur.de }}$

\section{Abstract}

A highly potent donor-acceptor biaryl TADF dye is accessible by a concise two-step sequence, employing twofold Ullmann arylation and a sequentially Pd-catalyzed Masuda borylationSuzuki arylation (MBSA). Photophysical investigations show efficient TADF at ambient temperature due to the sterical hindrance between the donor and acceptor moieties. The photoluminescence quantum yield amounts to $\Phi_{P L}=80 \%$ in toluene and $90 \%$ in PMMA arising from prompt and delayed fluorescence with decay times of $21 \mathrm{~ns}$ and $30 \mu \mathrm{s}$, respectively. From an Arrhenius plot, the energy gap $\Delta \mathrm{E}\left(\mathrm{S}_{1}-\mathrm{T}_{1}\right)$ between the lowest excited singlet $\mathrm{S}_{1}$ and triplet $\mathrm{T}_{1}$ state was determined to $980 \mathrm{~cm}^{-1}(120 \mathrm{meV})$. A new procedure is proposed that allows us to estimate the intersystem crossing time to $\approx 10^{2} \mathrm{~ns}$.

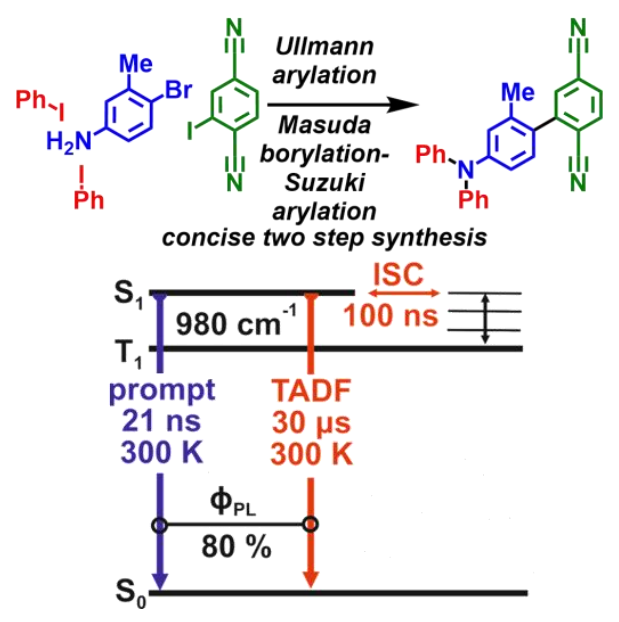

Organic light-emitting diodes (OLED) have considerably revolutionized modern illumination technology due to the involvement of phosphorescent emitter materials. By use of these, the limitation of usual fluorescent singlet emitters by the inherent 3:1 triplet:singlet exciton ratio 
could be overcome. Accordingly, efficient OLEDs could be developed. Meanwhile, they have reached a stage of commercial application from small smart phone to large tv displays. The emitters usually applied so far are based on phosphorescent materials., ${ }^{1,23}$ Currently, iridium(III) complexes are the best emitter materials to achieve this task. ${ }^{4,5}$ However, they are not well suited as stable blue-light emitters resulting in a low device stability. Moreover, these nobel metal complexes are rare and expensive. ${ }^{[1]}$ Thus, intense research activities have been initiated, either towards the cheaper $\mathrm{Cu}(\mathrm{I})$ or $\mathrm{Ag}(\mathrm{I})$ complexes $^{4,6,7,8}$ or metal-free organic materials $^{9,10,11,12,13,14}$, both classes employing the TADF (thermally activated delayed fluorescence) effect as a rationally designed singlet harvesting mechanism. ${ }^{15}$

Employing TADF (or "E-type emission") ${ }^{16}$ for exciton harvesting, almost $100 \%$ internal OLED quantum yield can be obtained. ${ }^{17}$ For achieving high TADF efficiency, a small energy gap between the excited singlet and the triplet state $\Delta E\left(S_{1}-T_{1}\right)$ is a prerequisite for an effective thermal up-ISC (or reverse ISC, RISC) from $\mathrm{T}_{1}$ to $\mathrm{S}_{1}{ }^{6,18,19}$ Twisted donor-acceptor systems are very promising lead structures with respect to small HOMO-LUMO overlap and thus, small $\Delta \mathrm{E}\left(\mathrm{S}_{1}-\mathrm{T}_{1}\right) \cdot{ }^{17,20,21,22}$

Based upon our program to develop sequential palladium-catalyzed one-pot methods for synthesizing functional organic chromophores, ${ }^{23}$ we decided to use the Masuda borylationSuzuki arylation (MBSA) one-pot process ${ }^{24}$ as a key step to access predicted donor-acceptor biaryl structures. The MBSA sequence represents an efficacious, fast and economical way to synthesize functional bi(hetero)aryl directly from the halogenated precursors. Here, we communicate a concise synthesis of a specific donor-acceptor biaryl structure and prove the TADF concept by TD-DFT calculations and detailed photophysical investigations. The resulting biaryl molecule represents an efficient and bright green emitting TADF compound.

The structure of the title compound 1 (Scheme 1) was proposed by using TD-DFT calculations with special regard to the energy gap $\Delta E\left(S_{1}-T_{1}\right)$ to achieve efficient TADF (see below). Therefore, a structure with an ortho-methyl group adjacent to the aryl-aryl-bond between the donor and acceptor parts was deduced, creating a twisting angle to obtain a reasonably small energy gap of the charge transfer (CT) states of singlet and triplet character.

Based upon transition metal catalyzed transformations, the target structure $\mathbf{1}$ was retrosynthetically analyzed by a cross-coupling of the bromotriarylamine 2 and 2-iodo terephthalicdinitrile 3 employing the MBSA sequence (Scheme 1). Bromotriarylamine 2 should be accessible by twofold Ullmann phenylation of the commercially available 4-bromo-3methylaniline 4 . 
<smiles>C=CC=Cc1ccc(C#N)cc1C(C)(C)c1ccc(N(c2ccccc2)c2ccccc2)cc1</smiles>

1<smiles>Cc1cc(N(c2ccccc2)c2ccccc2)ccc1Br</smiles>

2<smiles>N#Cc1ccc(C#N)c(I)c1</smiles>

3<smiles>C=CC=C</smiles>

Scheme 1. Retrosynthetic analysis of the calculated twisted biaryl TADF chromophore 1 by MBSA and Ullmann coupling.

Starting from commercially available 4-bromo-3-methylaniline 4 as the corner stone, the Ullmann reaction at $135^{\circ} \mathrm{C}$ with an excess of iodobenzene furnished the bromotriarylamine 2 in a yield of $77 \%$ (Scheme 2). This bromotriarylamine was then subjected to the MBSA sequence by first performing the Pd-catalyzed borylation with pinacolyl borane at $80^{\circ} \mathrm{C}$ in 1,4 dioxane in the presence of triethylamine for $3 \mathrm{~h}$ (monitored by GC-MS). After complete conversion of the bromide 2 to the corresponding pinalcolyl boronate, in the same reaction vessel methanol was added to quench excessive pinacolyl borane and for the Suzuki arylation 2-iodo terephthalic dinitrile 3 and cesium carbonate was added and the reaction mixture was heated to $100{ }^{\circ} \mathrm{C}$ for $11 \mathrm{~h}$ to furnish after chromatography the donor-acceptor biaryl 1 in $50 \%$ yield.

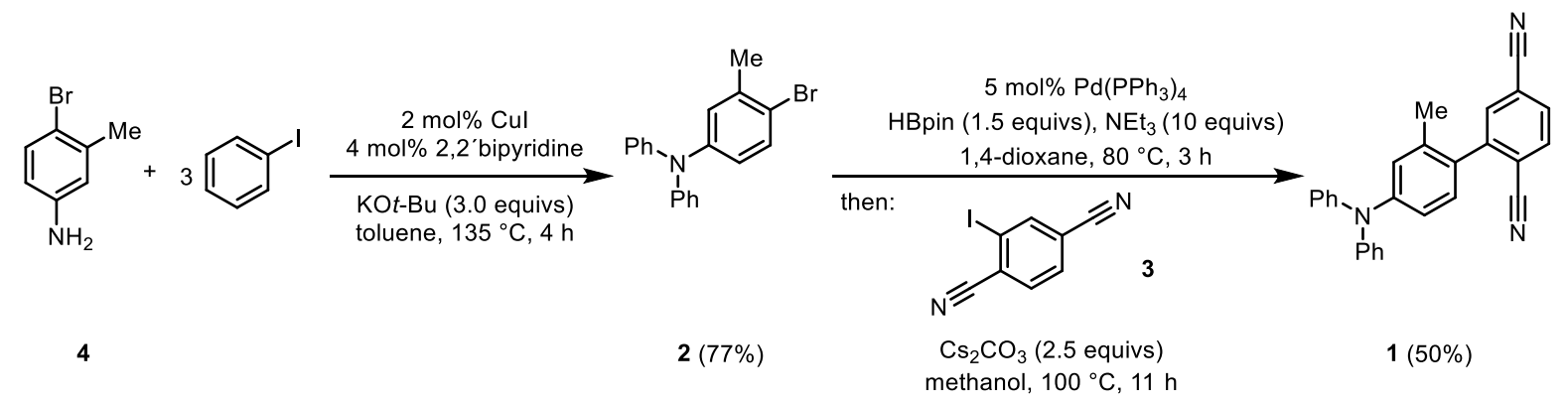

Scheme 2. Synthesis of the TADF chromophore 1. 
Frequently, a theoretical approach based on DFT and TD-DFT calculations give a good indication, whether a specific molecule will be a TADF candidate. Therefore, we carried out TD-DFT calculations for a DFT optimized molecule of compound 1. The calculations were done at the B3LYP/6-31G(d,p) level of theory ${ }^{25,26}$ for molecule 1 in a cavity of a toluene solvent and using the polarizable continuum model ${ }^{27}$ for inclusion of solvent effects.

The ground state optimized molecule 1 displays the dihedral angle between the planes of the donor and acceptor aromatic systems $\left(m-\left(\mathrm{Ph}_{2} \mathrm{~N}\right)\right.$-toluene and $p$-dicyanobenzene, respectively) of $\alpha=60^{\circ}$ (Figure 1). Obviously, the steric hindrance given by the methyl and cyano groups prevents a coplanar orientation of donor and acceptor fragments. At this conformation, the HOMO and LUMO frontier orbitals are well separated and localized the donor and acceptor fragment, respectively. Thus, the lowest electronic excitations have distinct charge-transfer (CT) character with an electron promoted from the donor's HOMO to the acceptor's LUMO. Indeed, TD-DFT calculations indicate a relatively small energy separation of $\triangle \mathrm{E}\left({ }^{1} \mathrm{CT}\left(\mathrm{S}_{1}\right)\right.$ $\left.{ }^{3} \mathrm{CT}\left(\mathrm{T}_{1}\right)\right)=840 \mathrm{~cm}^{-1}(0.1 \mathrm{eV})$. Both the $\mathrm{S}_{1}$ and $\mathrm{T}_{1}$ states result essentially from the $\mathrm{HOMO} \rightarrow$ LUMO transition and thus, HOMO and LUMO correspond well to natural transition orbitals, i. e. to "hole" and "electron", respectively.

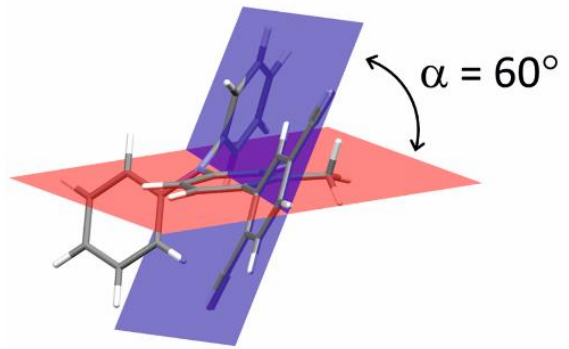

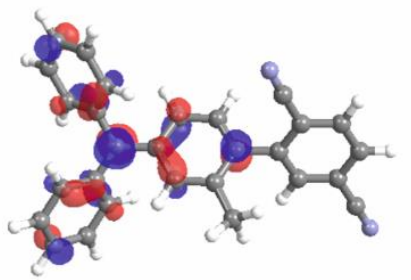

hole

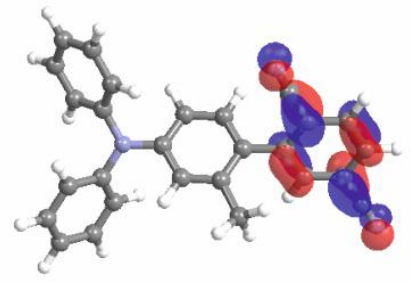

electron

Figure 1. Molecular geometry of compound 1 resulting from DFT calculations (B3LYP/3$31 \mathrm{G}(\mathrm{d}, \mathrm{p})$; solvent (toluene) modeled according to the polarizable continuum model (PCM) (compare Supplementary Information SI-7). The dihedral angle between the donor and acceptor planes is marked. At the right hand side the contour plots of natural transition orbitals $^{[28]}$ for the ground state $\mathrm{S}_{0} \rightarrow{ }^{1} \mathrm{CT}\left(\mathrm{S}_{1}\right)$ excitation are displayed. "Hole" and "electron" correspond essentially to HOMO and LUMO of the non-excited molecule, respectively.

The donor-acceptor compound 1 shows a bright green emission with $\lambda_{\max }(300 \mathrm{~K})=492 \mathrm{~nm}$ at a photoluminescence quantum yield of $\Phi_{\mathrm{PL}}=80 \%$ as determined for a degassed toluene solution at ambient temperature (PMMA (poly(methyl methacrylate) with $\approx 1 \%$ doping concentration of compound 1: $\left.\Phi_{\mathrm{PL}}=95 \%\right)$. Figure 2 a reproduces the steady state $(\mathrm{cW})$ emission spectrum exhibiting a halfwidth of about $3400 \mathrm{~cm}^{-1}(420 \mathrm{meV})$. Such a broad band width is expected for a donor to acceptor transition of CT character as predicted by the TDDFT calculations. Moreover, the observed red shift of the emission spectrum with increasing solvent polarity in the series of toluene, tetrahydrofurane to dichloromethane from $\lambda_{\max }(300 \mathrm{~K})$ $=492$ to $592 \mathrm{~nm}$ by $\approx 100 \mathrm{~nm}\left(\approx 3400 \mathrm{~cm}^{-1}\right)$ supports the CT-assignment of the lowest excited 
states. This indicates already a suitable situation for the occurrence of a small energy separation between the ${ }^{1} \mathrm{CT}\left(\mathrm{S}_{1}\right)$ and ${ }^{3} \mathrm{CT}\left(\mathrm{T}_{1}\right)$ states, being a condition for achieving TADF behavior.
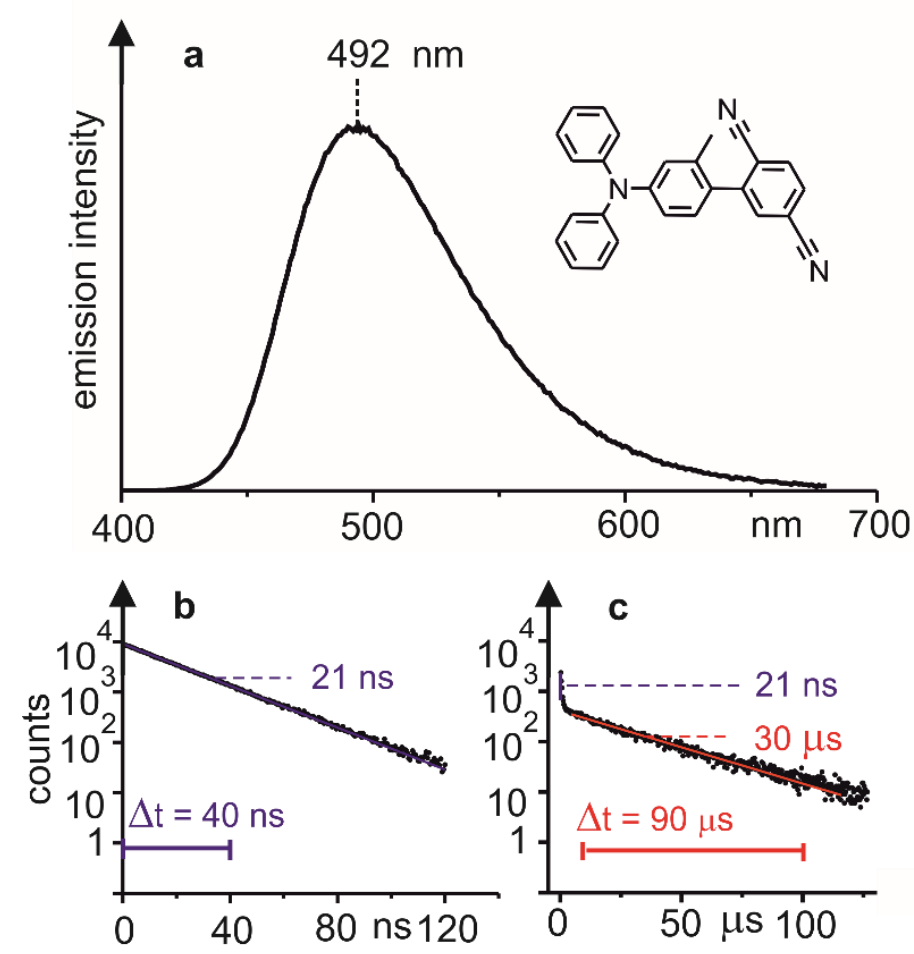

Figure 2. Luminescence properties of compound 1 recorded in degassed toluene at $300 \mathrm{~K}, \mathrm{c}$ $\approx 10^{-5} \mathrm{~mol} / \mathrm{L}$. a Time-integrated $(\mathrm{cw})$ emission spectrum excited at $\lambda_{\mathrm{exc}}=378 \mathrm{~nm}$. b Emission decay of the prompt fluorescence. Pulsed excitation at $\lambda_{\text {exc }}=378 \mathrm{~nm}, \lambda_{\text {det }}=492 \mathrm{~nm}$, pulsewidth $<100$ ps. c TADF decay (30 $\mu$ s component). Excitation at $\lambda_{\text {exc }}=378 \mathrm{~nm}$ with a rectangular pulse of a width of $50 \mu$ s to guarantee thermal equilibration (decay flank $\approx 10 \mathrm{~ns}$ ), $\lambda_{\text {det }}=492 \mathrm{~nm}$. (Supplementary Information is available in SI-8.)

The emission spectrum shown in Figure $2 \mathrm{a}$ is composed of two different components, a fast decaying one with a decay time of $21 \mathrm{~ns}$ and a longer decaying one of $30 \mu$ s (Figure $2 \mathrm{~b}$ and c). The short-lived component is assigned to the prompt fluorescence from the singlet charge transfer state ${ }^{1} \mathrm{CT}\left(\mathrm{S}_{1}\right)$ to the electronic ground state $\mathrm{S}_{0}$. Interestingly, this short decay time observed for compound $\mathbf{1}$ is significantly longer than found for typical $\pi^{*} \rightarrow \pi$ emitters. For these, the prompt fluorescence decay times usually lie in the range of 1 to $5 \mathrm{~ns}^{29}$ given by a significant allowedness of the corresponding transitions. The weaker allowedness (longer radiative decay time) of the ${ }^{1} \mathrm{CT}\left(\mathrm{S}_{1}\right) \rightarrow \mathrm{S}_{0}$ transition found for compound 1 can be tracked back to the small HOMO-LUMO overlap (Figure 1) and hence, the resulting small transition dipole moment. 6a,30 On the other hand, the long-lived component of $30 \mu$ s decay time is assigned as TADF emission. An alternative interpretation assigning this emission to result from triplet-triplet annihilation ${ }^{31}$ can be excluded due to the very low concentration of the compound and the very 
low excitation intensities applied during measurements. Further support for the TADF classification is given by its temperature dependence (see below).

Figure 3 reproduces ambient temperature time-resolved emission spectra. The prompt fluorescence spectrum is recorded without time delay after the laser pulse, i.e. $t=0 \mathrm{~ns}$, and a time window of $\Delta t=40 \mathrm{~ns}$ (Figure $2 \mathrm{~b}$ ), while the delayed spectrum is measured from $t=10 \mu \mathrm{s}$ with a time window of $\Delta t=90 \mu \mathrm{s}$ (Figure 2c). It is an important finding that both spectra shown in Figure 3 overlap almost completely. Exactly, this is expected for emissions that stem from the same ${ }^{1} \mathrm{CT}\left(\mathrm{S}_{1}\right)$ state, independently of prompt or delayed population. Thus, the delayed spectrum is clearly classified as TADF. An alternative assignment as phosphorescence can be excluded, since (i) a phosphorescence spectrum should appear at lower energy due to the singlet-triplet splitting of $980 \mathrm{~cm}^{-1}(120 \mathrm{meV}$ ) (see below) and (ii) the phosphorescence decay time, measured in toluene at $T=77 \mathrm{~K}$ with $\tau$ (phos $)=1.3 \mathrm{~s}\left(\Phi_{\mathrm{PL}}(\right.$ total $\left.)=100 \%\right)$ is more than four orders of magnitude longer than the observed decay of $\tau(\mathrm{TADF})=30 \mu \mathrm{s}$. The relatively fast TADF decay path (compared to the $T_{1} \rightarrow S_{0}$ paths) prevails competitive non-radiative quenching, hence, a high quantum yield of $\Phi_{\mathrm{PL}}=80 \%$ is found, even at ambient temperature and in solution.

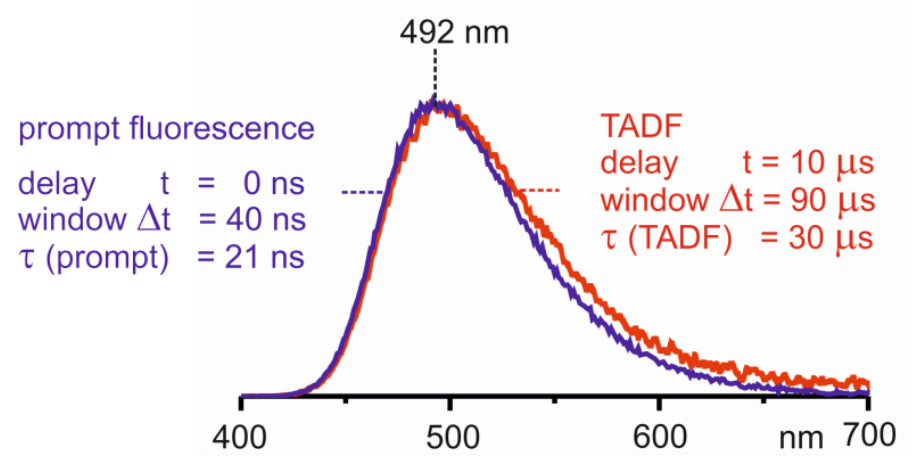

Figure 3. Time-resolved emission spectra of the prompt fluorescence measured with a time window from 0 to $40 \mathrm{~ns}$ and TADF detected after a delay time of $10 \mu \mathrm{s}$ with a time window of $90 \mu \mathrm{s}$ in degassed toluene at $300 \mathrm{~K}$. Pulsed excitation at $\lambda_{\mathrm{exc}}=378 \mathrm{~nm}$, pulse width $<100 \mathrm{ps}$ for the prompt fluorescence and $50 \mu$ width (rectangular pulse with a steep decay flank) for the delayed TADF component.

Further details of the TADF properties of compound 1 can be obtained by investigating the emission decay time of the long-living component in the temperature range between $T=200$ $\mathrm{K}$ and $300 \mathrm{~K}$. (Figure 4) In this range, toluene is liquid and the decay behavior is largely monoexponential. (Figure 4, inset) With temperature increase, the decay time continuously decreases from $1 \mathrm{~ms}(200 \mathrm{~K})$ to $30 \mu \mathrm{s}(300 \mathrm{~K})$, while the emission intensity is not changing significantly. Therefore, the decrease of the emission decay time is mainly attributed to an 
increase of the radiative rate with increasing temperature, which is induced by the activation of the decay path via the ${ }^{1} \mathrm{CT}$ state, which represents the TADF process.

According to the mono-exponential decay of the long-living component, thermal equilibration between the involved ${ }^{1} \mathrm{CT}$ and ${ }^{3} \mathrm{CT}$ states can be assumed to be established. This is valid at least after about $1 \mu$ s, i.e. after a time being much longer than the processes of prompt fluorescence and inter-system crossing (ISC). (See ref. 32 p. 194) In this situation, a Boltzmann-type population distribution of the involved states is valid. Hence, for an estimate, the experimentally accessible emission decay rate $k(\exp )=1 / \tau(\exp )$ can in analogy to refs 6 and 33 be expressed by eq. (1).

$$
k(\exp )=\frac{3 k\left(T_{1}\right)+k\left(S_{1}, I S C\right) \exp \left(-\frac{\Delta E\left(S_{1}-T_{1}\right)}{k_{B} T}\right)}{3+\exp \left(-\frac{\Delta E\left(S_{1}-T_{1}\right)}{k_{B} T}\right)}
$$

Herein $k\left(T_{1}\right)$ is the decay rate of the triplet emission and $k_{B}$ is the Boltzmann constant. $k\left(S_{1}\right.$, ISC) is the effective in-series-rate of the processes of ISC with $k$ (ISC) and prompt $S_{1} \rightarrow S_{0}$ emission with $k\left(S_{1}\right)$. An expression for $k\left(S_{1}, I S C\right)$ is obtained by solving the corresponding rate equations. (See SI-6) A value can be estimated from an extrapolation of experimental data as shown below. With this value, an approximate expression for $k$ (ISC) can be given (see Supplementary Information SI-6, eq. (S-16)):

$k(I S C)=k\left(S_{1}, I S C\right) \frac{k\left(S_{1}\right)-k\left(S_{1}, I S C\right)}{k\left(S_{1}\right)-2 k\left(S_{1}, I S C\right)}$

An alternative approach is mentioned in ref 34.

Eq. (1) can drastically be simplified if applied to the emission properties of compound 1. In particular, the energy separation $\Delta E\left(\mathrm{~S}_{1}-\mathrm{T}_{1}\right)>k_{B} T$. Below, it will be shown that $\Delta E\left(\mathrm{~S}_{1}-\mathrm{T}_{1}\right)$ amounts to $980 \mathrm{~cm}^{-1}$, while $k_{B} T$ at $300 \mathrm{~K}$ is $210 \mathrm{~cm}^{-1}$. Further, $k\left(\mathrm{~T}_{1}\right) \ll k\left(\mathrm{~S}_{1}, \mathrm{ISC}\right) . k\left(\mathrm{~T}_{1}\right)$ and $k\left(\mathrm{~S}_{1}, \mathrm{ISC}\right)$ are of the order of $1 \mathrm{~s}^{-1}$ and $10^{7} \mathrm{~s}^{-1}$, respectively (see below). Accordingly, eq. (1) simplifies in the logarithmic form to an Arrhenius-type equation

$$
\ln k(\exp )=\mathrm{a}-\frac{\Delta E\left(S_{1}-T_{1}\right)}{k_{B} T}
$$

This simple expression with $\mathrm{a}=\ln \left(\mathrm{k}\left(\mathrm{S}_{1}, \mathrm{ISC}\right) / 3\right)$ is applied to the experimental data as displayed in Figure 4. The slope of the straight line gives the energy separation of $\Delta E\left(\mathrm{~S}_{1}-\mathrm{T}_{1}\right)=$ $980 \mathrm{~cm}^{-1}(120 \mathrm{meV})$. This value is in good accordance to the calculated TD-DFT result of 840 $\mathrm{cm}^{-1}(104 \mathrm{meV})$ and fits well to the described TADF behavior. 


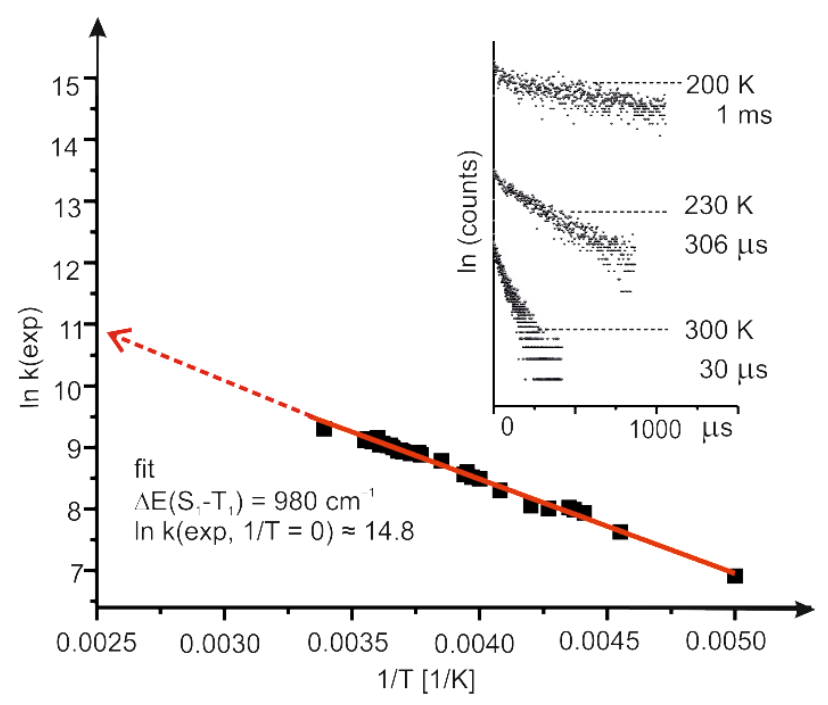

Figure 4. Arrhenius (Boltzmann) plot of the long-lived emission (TADF) component measured for compound 1 dissolved in toluene $\left(c \approx 10^{-5} \mathrm{~mol} / \mathrm{L}\right)$ for the temperature range of 200 to 300 $\mathrm{K}$. The red line represents a linear fit to the experimental data. By use of eq. $3, \Delta E\left(S_{1}-T_{1}\right)$ is determined $980 \mathrm{~cm}^{-1}(120 \mathrm{meV})$. The inset shows the decay time behavior at different temperatures. The short components caused by fluorescence in the time range up to several $100 \mathrm{~ns}$ were not taken into account. Pulsed excitation at $\lambda_{\text {exc }}=378 \mathrm{~nm}$, pulse width $50 \mu \mathrm{s}$ (rectangular pulse with a steep decay flank), $\lambda_{\text {det }}=492 \mathrm{~nm}$.

Moreover, the ISC time can very roughly be estimated from eq. (2). The value obtained by an extrapolation of the straight line to $1 / T=0$ as displayed in Figure 4 and using the approximation of eq. (3) gives $\ln k(\exp , 1 / T=0) \approx \ln \left(k\left(S_{1}, I S C\right) / 3\right) \approx 14.8 .{ }^{35}$ With this result and roughly approximating $k\left(\mathrm{~S}_{1}\right)^{-1}$ by the measured decay time of $21 \mathrm{~ns}$, we can estimate the magnitude of the ISC time constant to $\tau\left(\right.$ ISC) $\approx 10^{2} \mathrm{~ns}$.

For completeness, it is mentioned that the process of ISC between electronic states of the same configuration, i.e. between pure ${ }^{1} \mathrm{CT}$ and ${ }^{3} \mathrm{CT}$ states is formally forbidden, since these states do not exhibit spin-orbit coupling (SOC) between each other. ${ }^{36,37,38}$ But higher lying singlet and triplet state admixtures can slightly modify the character of the ${ }^{1,3} \mathrm{CT}$ states and thus, can lead to a slight allowedness for ISC. According to our TD-DFT calculations, adequate states of other configuration are found at about $0.5 \mathrm{eV}$ higher energy than the ${ }^{1,3} \mathrm{CT}$ states. Moreover, as recently discussed in detail, also vibronic coupling can induce distinct ISC..$^{39,40}$ However, a quantitative approach is not in the scope of this investigation.

Figure 5 summarizes the experimental results and fit data in an energy level diagram displaying also decay values. For comparison, we also add $77 \mathrm{~K}$ emission data for the phosphorescence as determined for compound $\mathbf{1}$ dissolved/doped in frozen/rigid toluene. The freezing process leads to an increase of the phosphorescence decay time and the emission quantum yield to 
$\tau($ phos $)=1.3 \mathrm{~s}$ and $\Phi_{\mathrm{PL}}=100 \%$, respectively. Moreover, freezing induces, as is well known, ${ }^{16}$ a blue shift of the steady state emission from $\lambda_{\max }(300 \mathrm{~K})=492 \mathrm{~nm}$ to $\lambda_{\max }(77 \mathrm{~K})=450 \mathrm{~nm}$.

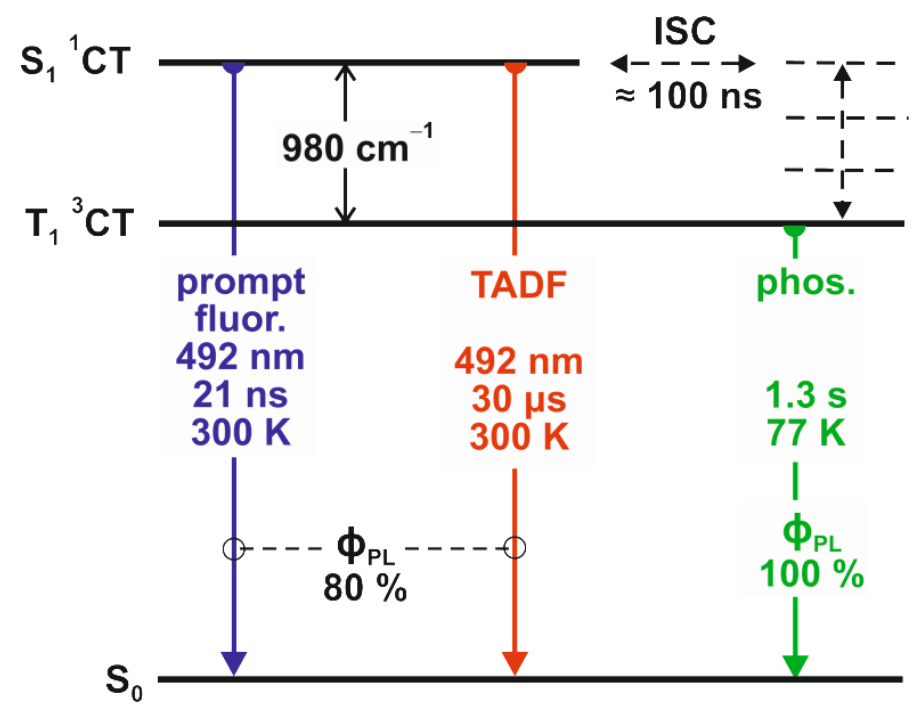

Figure 5. Schematic energy level diagram for compound 1 dissolved in toluene. The different emission mechanisms and corresponding decay times are indicated for $300 \mathrm{~K}$ and $77 \mathrm{~K}$, respectively. The intersystem crossing (ISC) time and the energy separation are determined from an Arrhenius plot. The vibrational/phonon levels (broken lines) are only indicated schematically.

A novel design concept for efficient TADF molecules based on conformationally distorted donor-acceptor systems was demonstrated to be successful. A new material, showing bright green emission with $\Phi_{\mathrm{PL}}=90 \%$ in PMMA (80\% in toluene) at a TADF decay time of $30 \mu \mathrm{s}$ at ambient temperature, was readily synthesized by applying the sequentially Pd-catalyzed MBSA procedure in very concise synthetic one-vessel routes. The resulting molecule exhibits favorable material properties for OLED fabrication, such as processibility by spin coating or sublimation. Increasing the rotational barriers between donor and acceptor by inducing more distinct sterical hindrances ${ }^{20 b}$ will not only allow for color tuning, but will lead to smaller singlettriplet splittings and thus, to shorter TADF decay times. Other types of chemical bonding between donor and acceptor are currently under investigation. For example, by strongly reducing the donor-acceptor interaction by minimizing residual donor-acceptor hyperconjugation, $\Delta E\left(S_{1}-T_{1}\right)<10 \mathrm{~cm}^{-1}(1 \mathrm{meV})$ values are obtainable. At this small energy separation, thermal activation is not a key property. All excitons are transferred/harvested directly to/in the lowest singlet state (Direct Singlet Harvesting Mechanism). This material concept will lead us to beyond TADF and might successfully be applied for next generation OLEDs. ${ }^{41,42}$ Novel molecular systems of this type and novel biaryl TADF dyes are currently under investigation. 


\section{Acknowledgments}

We cordially thank Fonds der Chemischen Industrie and Deutsche Forschungsgemeinschaft (Mu 1088/9-1) for the financial support. Moreover, we acknowledge financial funding from the German Ministery of Education and Research (BMBF). RC thanks for the financial support from the European Union's Horizon 2020 research and innovation programme under the Marie Skłodowska-Curie grant agreement No. 645628.

\section{Supplementary Information}

Chemical syntheses and analyses data, derivation of rate equations, experimental and computational details.

(1) Ogawa, S. (ed.) Organic Electronic Materials and Devices; Springer Japan: Tokyo 2015.

(2) Buckley, A. (ed.) Organic Light-Emitting Diodes (OLEDs): Materials, Devices and Applications; Woodhead Publishing: Oxford 2013.

(3) Yersin, H. (ed.) Highly Efficient OLEDs with Phosphorescent Materials; Wiley-VCH: Weinheim 2008.

(4) Yersin, H.; Rausch, A. F.; Czerwieniec, R.; Hofbeck, T.; Fischer, T. The Triplet State of Organotransition Metal Compounds. Triplet Harvesting and Singlet Harvesting for Efficient OLEDs, Coord. Chem. Rev. 2011, 255, 2622-2652.

(5) Zysman-Colman, E. (ed.) Iridium(III) in Optoelectronic and Photonic Applications, John Wiley \& Sons: Chichester (UK) 2017.

(6) Czerwieniec, R.; Leitl, M. J.; Homeier, H. H. H.; Yersin, H. Cu(I) Complexes - Thermally Activated Delayed Fluorescence. Photophysical Approach and Material Design. Coord. Chem. Rev. 2016, $325,2-28$.

(7) Shafikov, M. Z.; Suleymanova, A. F.; Czerwieniec, R.; Yersin, H. Design Strategy for Ag(I)Based Thermally Activated Delayed Fluorescence Reaching an Efficiency Breakthrough. Chem. Mater. 2017, 29, 1708 - 1715.

(8) Chen, J.; Teng, T.; Kang, L.; Chen, X.-L.; Wu, X.-Y.; Yu, R.; Lu, C.-Z. Highly Efficient Thermally Activated Delayed Fluorescence in Dinuclear Ag(I) Complexes with a Bis-Bidentate Tetraphosphane Bridging Ligand. Inorg. Chem. 2016, 55, 9528-9536.

(9) Uoyama, H.; Goushi, K.; Shizu, K.; Nomura, H.; Adachi, C. Highly Efficient Organic Light-emitting Diodes from Delayed Fluorescence. Nature 2012, 492, 234-238.

(10) Hosokai, T.; Matsuzaki, H.; Nakanotani, H.; Tokumaru, K.; Tsutsui, T.; Furube, A.; Nasu, K.; Nomura, H.; Yahiro, M.; Adachi, C. Evidence and Mechanism of Efficient Thermally Activated Delayed Fluorescence Promoted by Delocalized Excited States. Sci. Adv. 2017, 3, e1603282.

(11) Cui, L.-S.; Nomura, H.; Geng, Y.; Uk Kim, J.; Nakanotani, H.; Adachi, C. Controlling SingletTriplet Energy Splitting for Deep-Blue Thermally Activated Delayed Fluorescence Emitters. Angew. Chem. Int. Ed. 2017, 56,1571-1575.

(12) Chen, X.-L.; Jia, J.-H.; Yu, R.; Liao, J.-Z.; Yang, M.-X.; Lu, C.-Z. Combining Charge-Transfer Pathways to Achieve Unique ThermallyActivated Delayed Fluorescence Emitters for HighPerformanceSolution-Processed, Non-doped Blue OLEDs. Angew. Chem. Int. Ed. 2017, $56,15006-15009$.

(13) Wong, M. Y.; Zysman-Colman, E. Purely Organic Thermally Activated Delayed Fluorescence Materials for Organic Light-Emitting Diodes Adv. Mater. 2017, 29, 1605444..

(14) Komatsu, R.; Ohsawa, T.; Sasabe, H.; Nakao, K.; Hayasaka, Y.; Kido, Manipulating the Electronic Excited State Energies of Pyrimidine-Based Thermally Activated Delayed Fluorescence Emitters To Realize Efficient Deep-Blue Emission. J.; ACS Appl. Mater. Interfaces 2017, 9, 4742-4749

(15) a) Czerwieniec, R.; Yu, J.; Yersin, H. Blue-Light Emission of $\mathrm{Cu}(\mathrm{I})$ Complexes and Singlet Harvesting. Inorg. Chem. 2011, 50, 8293 - 8301. b) Yersin, H.; Monkowius, U.; Komplexe mit 
kleinen Singulett-Triplett-Energie-Abständen zur Verwendung in Opto-elektronischen Bauteilen (Singulett-Harvesting-Effekt). DE 102008 033563, 2008.

(16) Parker, C. A.; Hatchard, C. G. Triplet-singlet emission in fluid solutions. Trans. Faraday Soc. 1961, 57, $1894-1904$.

(17) Kaji, H.; Suzuki, H.; Fukushima, T.; Shizu, K.; Suzuki, K.; Kubo, S.; Komino, T.; Oiwa, H.; Suzuki, F.; Wakamiya, A.; Murata, Y.; Adachi, C. Purely Organic Electroluminescent Material Realizing 100\% Conversion from Electricity to Light. Nat. Commun. 2015, 6, 8476.

(18) Endo, A.; Ogasawara, M.; Takahashi, A.; Yokoyama, D.; Kato, Y.; Adachi, C. Thermally Activated Delayed Fluorescence from Sn(IV)-Porphyrin Complexes and Their Application to Organic LightEmitting Diodes - A Novel Mechanism for Electroluminescence. Adv. Mater. 2009, 21, $4802-$ 4806.

(19) Li, M.; Liu, Y.; Duan, R.; Wei, X.; Yi, Y.; Wang, Y.; Chen, C. F. Aromatic-Imide-Based Thermally Activated Delayed Fluorescence Materials for Highly Efficient Organic Light-Emitting Diodes. Angew. Chem. Int. Ed. 2017, 56, 8818 -8822.

(20) Mataranga-Popa, L. N.; Czerwieniec, R.; Yersin, H. Organic Molecule Having Small TripletSinglet Energy Spacings for Effective Delayed Fluorescence for Application in Optoelectronic Devices. Patent application 2014, DE 102014101920.2 and WO 2015/121239 A1. b) Mataranga-Popa, L. N.; Czerwieniec, R.; Yersin, H.; Organic TADF Molecules Having Steric Hindrance at the Donor and at the Acceptor Optoelectronic Devices. Patent application 2014, DE 102014101921.0 and WO 2015/121241 A1.

(21) Lee, S. Y.; Yasuda, T.; Park, I. S.; Adachi, C. X-shaped Benzoylbenzophenone Derivatives with Crossed Donors and Acceptors for Highly Efficient Thermally Activated Delayed Fluorescence Dalton Trans. 2015, 44, 8356-8359.

(22) Suzuki, K.; Kubo, S.; Shizu, K.; Fukushima, T.; Wakamiya, A.; Murata, Y.; Adachi, C.; Kaji, H. Triarylboron-Based Fluorescent Organic Light-Emitting Diodes with External Quantum Efficiencies Exceeding 20 \%. Angew. Chem. Int. Ed. 2015, 54, 15231-15235.

(23) Levi, L.; Müller, T. J. J. Multicomponent Syntheses of Functional Chromophores. Chem. Soc. Rev. 2016, 45, 2825-2846.

(24) a) Tasch, B. O. A.; Bensch, L.; Antovic, D.; Müller, T. J. J. Masuda Borylation-Suzuki Coupling (MBSC) Sequence of Vinylhalides and its Application in a One-pot Synthesis of 3,4Biarylpyrazoles. Org. Biomol. Chem. 2013, 11, 6113-6118. b) Tasch, B. O. A.; Antovic, D.; Merkul, E.; Müller. T. J. J. One-Pot Synthesis of Camalexins and 3,3'-Biindoles by the Masuda BorylationSuzuki Arylation (MBSA) Sequence. Eur. J. Org. Chem. 2013, 4564-4569. c) Merkul, E.; Schäfer, E.; Müller, T. J. J. Rapid Synthesis of Bis(hetero)aryls by One-pot Masuda borylation-Suzuki Coupling Sequence and its Application to Concise Total Syntheses of Meridianins A and G. Org. Biomol. Chem. 2011, 9, 3139-3141. d) Baudoin, O.; Guénard, D.; Guéritte, F. PalladiumCatalyzed Borylation of Ortho-Substituted Phenyl Halides and Application to the One-Pot Synthesis of 2,2'-Disubstituted Biphenyls. J. Org. Chem. 2000, 65, 9268-9271.

(25) Becke, A.D. Density-functional Thermochemistry. III. The Role of Exact Exchange. J. Chem. Phys. 1993, 98, 5648-5652.

(26) Petersson, G. A.; Al-Laham, M. A. A Complete Basis Set Model Chemistry. II. Open-shell Systems and the Total Energies of the First-row Atoms. J. Chem. Phys., 1991, 94, 6081-90.

(27) Tomasi, J.; Mennucci, B.; Cammi, R. Quantum Mechanical Continuum Solvation Models. Chem. Rev., 2005, 105, 2999-3093.

(28) Martin, R. L. Natural Transition Orbitals. J. Chem. Phys., 2003, 118, 4775-4777

(29) Turro, N. J. Modern Molecular Chemistry. Bejamin/Cummings, Menlo Park, California 1978.

(30) Yersin, H.; Czerwieniec, R.; Shafikov, Z.; Suleymanova, A. F. TADF Material Design. Photophysical Background and Case Studies Focusing on $\mathrm{Cu}(\mathrm{I})$ and $\mathrm{Ag}(\mathrm{I})$ Complexes. Chem. Phys. Chem. 2017, 18, 3508 - 3535.

(31) Birks, J. B. Photophysics of Aromatic Molecules. Wiley-Interscience: London 1970.

(32) Yersin, H.; Humbs, W.; Strasser, J. Characterization of Excited Electronic and Vibronic States of Platinum Metal Compounds with Chelate Ligands by Highly Frequency-resolved and Timeresolved Spectra. Top. Curr. Chem. 1997, 191, 153 - 249.

(33) Azumi, T.; O'Donnell, C. M.; McGlynn, S. P. On the Multiplicity of the Phosphorescent State of Organic Molecules. J. Chem. Phys. 1966, 45, 2735-2742. 
(34) Interestingly, an alternative, very simple approach for the in-series-rate $\mathrm{k}(\mathrm{S} 1, \mathrm{ISC})$ according to $k(S 1, I S C)^{-1}=k(S 1)^{-1}+k(I S C)^{-1}$ results in almost the same ISC time as determined by use of the rate equations as presented in the Supporting Information SI-6.

(35) The measured data points correspond to temperatures where the exponential in the denominator of eq. (1) is close to zero and can be neglected with respect to 3 . Hence, we obtain eq. (3). But extrapolation to $1 / T=0$ corresponds to $\exp (0)=1$ for this exponential. Thus, we cannot expect more than a rough estimate.

(36) El-Sayed, M. A. Spin-Orbit Coupling and the Radiationless Processes in Nitrogen Heterocyclics. J. Chem. Phys. 1963, 38, 2834-2838.

(37) Rausch, A. F.; Homeier, H. H. H.; Djurovich, P. I.; Thompson, M. E.; Yersin, H.; Spin-orbit Coupling Routes and OLED Performance - Studies of Blue-light Emitting $\operatorname{Ir}$ (III) and Pt(II) Complexes, Proc. of SPIE 2007, 6655, 66550F-1 - 66550F-16.

(38) Rausch, A. F.; Homeier, H. H. H.; Yersin, H. Organometallic Pt(II) and Ir(III) Triplet Emitters for OLED Applications and the Role of Spin-Orbit Coupling: A Study Based on High-Resolution Optical Spectroscopy. Top. Organomet. Chem. 2010, 29, 193-235.

(39) Marian, C. M.; Föller, J.; Kleinschmidt, M.; Etinski, M.; Intersystem Crossing Processes in TADF Emitters. In: Highly Efficient OLEDs. Materials Based on TADF, Yersin, H. (ed.); Wiley-VCH, Weinheim Germany 2018, DOI: 10.1002/9783527691722, in press

(40) Penfold, T. J.; Gibson, J.; The Role of Vibronic Coupling for Intersystem Crossing and Reverse Intersystem Crossing Rates in TADF Molecules. In: Highly Efficient OLEDs. Materials Based on TADF, Yersin, H. (ed.); Wiley-VCH, Weinheim Germany 2018, DOI: 10.1002/9783527691722, in press.

(41) Yersin, H.; Mataranga-Popa, L.; Czerwieniec, R.; Organische Moleküle für Direktes SingulettHarvesting mit kurzer Emissionsabklingzeit zur Verwendung in optoelektronischen Vorrichtungen. Europaen patent EP 17170682.3, 2017 and German patent DE 102017 101432.2, 2017.

(42) Yersin, H.; Mataranga-Popa, L.; Li, S.-W.; Czerwieniec, R. Design strategies for materials showing thermally activated delayed fluorescence and beyond: Towards the fourth-generation OLED mechanism. J. Soc. Info. Display. doi:10.1002/jsid.654. 